\title{
Genotype distribution of human papillomavirus (HPV) in histological sections of cervical intraepithelial neoplasia and invasive cervical carcinoma in Madrid, Spain
}

\author{
Benjamín García-Espinosa ${ }^{1}$, Ernesto Moro-Rodríguez ${ }^{1,3^{*}}$ and Emilio Álvarez-Fernández²
}

\begin{abstract}
Background: Human Papillomavirus (HPV) genotype distribution and co-infection occurrence was studied in cervical specimens from the city of Madrid (Spain), as a contribution to the knowledge of Human Papillomavirus genotype distribution and prevalence of carcinogenic HPV types in cervical lesions in Spain.

Methods: A total of 533 abnormal specimens, from the Hospital General Universitario "Gregorio Marañón" of Madrid, were studied. These included 19 benign lesions, 349 cervical intraepithelial neoplasias 1 (CIN1), 158 CIN2-3 and 7 invasive cervical carcinomas (ICC). HPV genotyping was performed using PCR and tube array hybridization.

Results: We detected 20 different HPV types: 13 carcinogenic high-risk HPV types (HR-HPVs), 2 probably carcinogenic high-risk HPV types (PHR-HPVs) and 5 carcinogenic low-risk HPV types (LR-HPVs). The most frequent HPV genotypes found in all specimens were HPV16 (26.0\%), 31 (10.7\%) and 58 (8.0\%). HPV 18 was only detected in 5.0\%. Co-infections were found in 30.7\% of CIN 1 and $18.4 \%$ cases of CIN2-3. The highest percentage of HR HPVs was found in those specimens with a CIN2-3 lesion (93.7\%).

Conclusion: As our study shows the current tetravalent vaccine could be effective in our geographical area for preventing all the invasive cervical carcinomas. In addition, upon the estimates of the important presence of other HR-HPV types - such as 31, 58, 33 and 52 - in different preneoplasic lesions the effectiveness of HPV vaccination in our geographical area, and others with similar genotype distribution, should be limited.
\end{abstract}

Keywords: Human papillomavirus, Polymerase chain reaction, Genotyping, Squamous intraepithelial lesions, Cervix, Spain

\section{Background}

Cervical cancer is the second most common cancer in women worldwide. Several epidemiological studies make it possible to conclude that persistent infection by certain types of HPV is a causal and necessary factor for the development of cervical cancer. A series of HPV-induced precursor lesions, from LSIL to HSIL, may lead to ICC. More than 40 anogenital HPV types exist [1]. From an epidemiological point of view, based on their association

\footnotetext{
* Correspondence: joseernesto.moro@urjc.es

'Department of Histology and Anatomical Pathology, Rey Juan Carlos

University School of Medicine, Madrid, Spain

${ }^{3}$ Universidad Rey Juan Carlos, Av de Atenas s/n., E28922 Alcorcón, Madrid, Spain

Full list of author information is available at the end of the article
}

with cervical cancer and precursor lesions, HPVs have been classified in two groups: high risk of carcinogenesis HPV types (HR-HPVs) and low risk of carcinogenesis HPV types (LR-HPVs) [2,3]. There are also two additional groups of probable high risk of carcinogenesis HPV types (PHR-HPVs) and indeterminate risk of carcinogenesis HPV (IR-HPVs) [4]. But even amongst the HR-HPV genotypes, the variation of their oncogenic potential is considerable, which may vary depending on specific intratypic HPV variations or on ethnic background and lifestyle factors of the human population under study.

HR-HPV 16 is the most common type in all studies, but there are some differences regarding its prevalence to other HPV types. HR-HPV 16 and 18 are estimated 
responsible for nearly $70 \%$ of cervical cancer cases worldwide. Co-infection with multiple HPV types could also increase the risk of cervical lesion. However, followup studies suggest that the presence of multiple types does not influence the course of HPV infections [5].

Two types of prophylactic vaccines have been developed to prevent cervical cancer: a bivalent vaccine against HR-HPV 16 and 18, and a tetravalent vaccine against HR-HPV 16/18 and LR-HPV 6 and 11 genotypes that are responsible for most genital warts [6-8].

Some studies suggest that these vaccines seem to protect against some HPV 16-related types (31, 33, 35 and 52) and HPV 18-related types (39, 45, 59, 68 and 85). However, vaccination against HPV 16/18 does not seem to cross-protect against HPV $58[9,10]$. In addition, the decreasing prevalence of the genotypes included in the HPV vaccine may increase the prevalence of other coinfecting genotypes. This hypothesis suggests that the elimination of certain genotypes by vaccination may affect the distribution of other genotypes, and the impact of the vaccine could vary [9].

Moreover, it has been demonstrated that the distribution of HPV 16 and 18 and the other HR-HPV genotypes vary around the world both in type and relative incidence. Therefore, the effect of HPV16/18 vaccines will, at least to some degree, vary by region. However, regional differences appear to become less pronounced with increasing severity of lesions, as HPV 16 becomes increasingly dominant [11].

The age-adjusted HPV prevalence in Spanish women from general population is very low (3\%) [12]. However, this prevalence increase to $34.4 \%$ in Spanish women with cytological alterations [13]. In addition, changes in sexual behaviour and human migration flows could contribute to introduce some degree of variation both in prevalence and distribution of HPV types [14]. Additional genotypes that are not included in the first generation of human papillomavirus vaccines are frequently associated with cervical cancer in Latin America and Africa [15]. Over the past decade a large number of immigrants have arrived in Spain; more or less $40 \%$ come from Latin America and 50\% are women, and the prevalence of HR-HPVs is more than three times higher in Latin Americans than in Spaniards [14]. Therefore, this significant immigration from Latin America, as well as from Eastern Europe and North Africa, possibly leads to the appearance in Spain of additional carcinogenic genotypes not included in current HPV vaccines $[16,17]$.

For all these reasons, it is important to estimate the prevalence of different HPV types found in cervical cancer in different geographic regions and over time in order to study the carcinogenic function of each genotype, to assess the impact of the current vaccines and to guide the introduction of a new generation of them $[13,18,19]$.
The regional community of Madrid is located in the centre of Spain and has a population of 6.4 million. In the last ten years, a high influx of immigrants from Latin America, Eastern Europe and North Africa has caused a population increase of nearly 15 per cent. The objective of this study is to provide epidemiological data regarding the prevalence and distribution of different HPV genotypes in samples with different grade of cervical lesions, obtained in our geographical area, in order to understand the carcinogenic potential of each of HPV type found and to discern the impact of HPV vaccination on our population.

\section{Methods}

\section{Specimen collection and diagnosis}

This is a cross-sectional and retrospective study, in which all cases diagnosed between January 2005 and July 2011 (1,137 abnormal specimens from fixed tissue sections of biopsies and LEEPs) were selected. Samples for HPV testing, containing cervical carcinoma related lesions, were collected by different departments of the Hospital General Universitario "Gregorio Marañón" of Madrid and submitted to the anatomical pathology laboratory of this hospital. In 533 of this specimens one or several HPV types was detected. The population included in this study had been selected for any type of gynaecologic pathology. This hospital provides healthcare to a population of about 750,000 individuals $(11.5 \%$ of the population of the regional community of Madrid).

The positive samples included 19 benign lesions (genital warts, condylomas and papillomas), 349 cervical intraepithelial neoplasias 1 (CIN1), 158 CIN2-3 and 7 invasive cervical carcinomas (ICC).

Informed consent was not required for this study since the results presented here come from HPV genotyping routinely performed, as an adjunct to the cytological and histological study, in an anatomical pathology laboratory. The detection and genotyping was done in clinical setting and in order to protect patient confidentiality the identifiers of personal data were always deleted, that is why it was not possible to determine the ethnic of each patient. The study was supervised by the ethical committee of our hospital (Comité ético de investigación clínica - CEIC).

\section{Detection and genotyping of HPV}

The DNA was obtained from fixed tissue sections of buffered formalin and embedded in paraffin (Kit for DNA extraction. Master Diagnostica SL Granada).

This DNA was used as a template to detect the presence of HPV DNA by PCR amplification using primers specific GP5-6 L1 consensus region (HPV screening kit. Master Diagnostica SL Granada). 
The result of the amplification was visualized by agarose gel electrophoresis and ethidium bromide staining on a UV transilluminator, considering the case as positive if it showed a band of $150 \mathrm{bp}$.

Among the HPV positive cases we identified the type of virus using a kit that allows the detection of 14 specific HPV types of high-risk (16, 18, 31, 33, 35, 39, 45, 51, 52, $56,58,59,68), 2 \mathrm{HPV}$ types of intermediate-risk $(53,66)$ of and 5 HPV types of low-risk $(6,11,42,43,44)$. This identification was performed by PCR amplification of a fragment of 450 bp L1 consensus region and reverse hybridization with probes specific for each type (HPV GenoArray Test Kit. Ref: IHP301-C. Master Diagnostica SL Granada. Supplier: HybriBio Limited, Hong Kong).

Two methods were used to estimate the frequency of HPV positivity: percentages referred to the number of lesions infected by one or several genotypes and percentages referred to the total number of virus detected in each kind of lesion and in the total of them.

\section{Statistical analysis}

Statistical analysis was performed using Stata version 11.1/SE (StataCorp. LP, TX, USA).

Relative frequencies of HPV genotypes were estimated as percentages and their 95\% confidence intervals were obtained with Clopper-Pearson method based on exact binomial distribution of tail areas.

\section{Results}

\section{Distribution of viral genotypes}

The percentage of HPV positivity in the total of specimens studied was at less than $47 \%(533 / 1,137)$.

Taking into account overall data, we detected 20 different HPV types: 13 HR-HPVs, 2 PHR-HPVs and 5 LR-HPVs.

HPV 16 was the most common type (26.0\%) followed, in order of decreasing frequency, by HPV 31 (10.7\%), HPV 58 (8.0\%), HPV 6 (6.2\%), HPV 33 (6.1\%), HPV 52 (5.7\%), HPV 53 (5.1\%), HPV 18 (5.0\%), HPV 66 (4.4\%) and HPV 11 (4.3\%).

HPV 16 was present in $35.3 \%$ of total lesions, HPV 31 in 14.4\%, HPV 58 in 10.9\%, HPV 6 in $8.4 \%$ and HPV 33 in $8.3 \%$ of them.
In the whole of lesions included in our study, $63.0 \%$ of HPVs detected were HR-HPVs, $12.2 \%$ were PHR-HPVs and $15.2 \%$ were LR-HPVs. HPVs 16 and/or 18 were founded in 41.3\% of lesions and HPVs 6 and 11 in 14.1\% of them.

Nine (1.2\%) of HPV-positive cases detected were classified as an uncharacterized type (HPV X), but it is likely that this classification was related with a missed detection of known types and was not associated to an infection by HPV types to be discovered (Table 1).

\section{Relationship between diagnoses and HPV genotypes}

In benign lesions, HPV 6 was the most common type (42.1\%) followed, in order of decreasing frequency, by HPV 11 (26.3\%), HPV 16 (15.8\%), HPV 18 (5.3\%) and HPV 53 (5.3\%).

$21.1 \%$ of HPVs detected were HR-HPVs, $5.3 \%$ were PHR-HPVs and 68.4\% were LR-HPVs. HPVs 16 and/or 18 comprised $21.1 \%$ of HPV infections detected and HPVs 6 and/or 11 comprised $68.4 \%$ of them. Therefore, LR-HPV types included in tetravalent vaccine were detected more frequently than viruses not included in this vaccine.

In CIN1 cases, HPV 16 was the most common type (18.2\%) followed, in order of decreasing frequency, by HPV 31 (12.0\%), HPV 58 (9.0\%), HPV 6 (6.4\%), HPV 53 (6.4\%), HPV 33 (6.0\%), HPV 66 (6.0\%), HPV 52 (5.2\%), HPV 18 (5.0\%) and HPV 68 (4.8\%).

HPV 16 was present in $26.1 \%$ of CIN1 lesions, HPV 31 in $17.2 \%$, HPV 58 in $12.9 \%$, HPV 53 in $9.2 \%$ and HPV 6 in $9.2 \%$ of them.

HR-HPVs were detected in 51.0\% of CIN1 lesions, PHR-HPVs in $16.6 \%$ and LR-HPVs in $16.3 \%$ of them. HPVs 16 and/or 18 were present in 32.4\% of lesions and HPVs 6 and/or 11 in $14.6 \%$ of them.

HR-HPV types not included in vaccines were detected more frequently (59.9\%) than carcinogenic viruses included in vaccines (32.4\%). LR-HPV types were infrequently identified as single infections (15.3\%).

In CIN2-3 cases, HPV 16 was the most common type (45.2\%) followed, in order of decreasing frequency, by HPV 31 (8.6\%), HPV 52 (7.6\%), HPV 33 (7.1\%), HPV 58 (6.6\%), HPV 18 (4.6\%), HPV 6 (2.5\%), HPV 11 (2.5\%), HPV 51 (2.5\%), HPV 53 (7.1\%) and HPV 68 (2.0\%).

Table 1 Frequency of HPV positivity according to the type of cervical lesion found

\begin{tabular}{lcccc}
\hline Pathological diagnosis & $\begin{array}{c}\text { Number (\%) } \\
\text { HPV + cases }\end{array}$ & $\begin{array}{c}\text { Number (\%) single } \\
\text { HPV cases }\end{array}$ & $\begin{array}{c}\text { Number (\%) multiple } \\
\text { HPV cases }\end{array}$ & $\begin{array}{c}\text { Number (\%) } \\
\text { HPV X cases }\end{array}$ \\
\hline Benign lesions & $19(3.6 \%)$ & $19(100 \%)$ & $0(0 \%)$ & $1(5.3 \%)$ \\
CIN 1 & $349(65.5 \%)$ & $242(69.3 \%)$ & $107(30.7 \%)$ & $5(1.4 \%)$ \\
CIN 2-3 & $158(29.6 \%)$ & $129(81.6 \%)$ & $29(18.4 \%)$ & $3(1.9 \%)$ \\
ICC & $7(1.3 \%)$ & $7(100 \%)$ & $0(0 \%)$ & $0(0 \%)$ \\
Total cases & $533(100 \%)$ & $397(74.5 \%)$ & $136(25.5 \%)$ & $9(1.7 \%)$ \\
\hline
\end{tabular}


HPV 16 was present in 56.3\% of CIN2-3 lesions, HPV 31 in 10.8\%, HPV 52 in 9.5\%, HPV 33 in $8.9 \%$ and HPV 58 in $8.2 \%$ of them.

HR-HPVs were detected in $93.7 \%$ of CIN2-3 lesions, PHR-HPVs in $3.8 \%$ and LR-HPVs in $6.3 \%$ of them. HPVs 16 and/or 18 were present in $61.4 \%$ of lesions and HPVs 6 and/or 11 in $6.3 \%$ of them.

HR-HPV types not included in vaccines were detected with an inferior frequency (42.4\%) to carcinogenic viruses included in vaccines (61.4\%). LR-HPV types were rarely identified as single infections (4.7\%).

In ICC cases, HPV 16 was the most common type (71.4\%) followed, in order of decreasing frequency, by HPV 18 (14.3\%) and HPV 11 (14.3\%).

HR-HPVs were detected in $85.7 \%$ of ICC lesions and LR-HPVs in $14.3 \%$ of them. PHR-HPVs were not detected in this kind of lesions. HPVs 16 and/or 18 were present in 85.7\% of lesions and HPVs 6 and/or 11 in 14.3\% of them.
The distribution of HPV types and the analysis between pathological groups vs. HPV risk types is shown in Tables 2 and 3.

\section{Genotypes found as co-infections}

The percentages of multiple infections were $30.7 \%$ in CIN1 cases and $18.4 \%$ in CIN2-3 cases. Multiple infections were not detected in benign lesions and in ICC.

In all sorts of lesions with multiple infections the most common pattern of co-infection was double infection with HPV 16 and 58 (6 cases), followed by double infection with HPV 16/31 (5 cases), HPV 16/53 (5 cases), HPV 16/18 (4 cases) and 16/52 (4 cases).

In CIN1 multiple infections cases, most co-infections consisted of two different genotypes (71.0\%). $20.6 \%$ of the cases showed triple genotype infection and only $6.5 \%$ of them showed quadruple genotype infection. Surprisingly, in one case $(0.9 \%)$ five different genotypes were

Table 2 Distribution of HPV genotypes found in the study according to the pathological diagnosis

\begin{tabular}{|c|c|c|c|c|c|c|c|c|c|c|c|c|c|c|c|}
\hline \multirow{2}{*}{$\begin{array}{l}\text { Genotype } \\
\text { found }\end{array}$} & \multicolumn{5}{|c|}{ Total lesions } & \multicolumn{5}{|c|}{ Benign lesions } & \multicolumn{5}{|c|}{ CIN 1} \\
\hline & $\mathbf{N}$ & $\% *$ & $\mathrm{Cl} 195 \%$ & $\% * *$ & $\mathrm{Cl} 95 \%$ & $\mathbf{N}$ & $\% *$ & $\mathrm{Cl} 95 \%$ & $\% * *$ & $\mathrm{Cl} 195 \%$ & $\mathbf{N}$ & $\% *$ & $\mathrm{Cl} 195 \%$ & $\% * *$ & $\mathrm{Cl} 195 \%$ \\
\hline \multicolumn{16}{|l|}{ HR-HPVs } \\
\hline 16 & 188 & 35.3 & $31.2-39.5$ & 26.0 & $22.9-29.4$ & 3 & 15.8 & $3.4-39.6$ & 15.8 & $3.4-39.6$ & 91 & 26.1 & $21.5-31.0$ & 18.2 & $14.9-21.9$ \\
\hline 18 & 36 & 6.8 & $4.8-9.2$ & 5.0 & $3.5-6.8$ & 1 & 5.3 & $0.1-26.0$ & 5.3 & $0.1-26.0$ & 25 & 7.2 & $4.7-10.4$ & 5.0 & $3.3-7.3$ \\
\hline 31 & 77 & 14.4 & $11.6-17.7$ & 10.7 & $8.5-13.1$ & 0 & 0 & $0.0-0.0$ & 0 & $0.0-0.0$ & 60 & 17.2 & $13.4-21.6$ & 12.0 & $9.3-15.2$ \\
\hline 33 & 44 & 8.3 & $6.1-10.9$ & 6.1 & $4.5-8.1$ & 0 & 0 & $0.0-0.0$ & 0 & $0.0-0.0$ & 30 & 8.6 & $5.9-12.0$ & 6.0 & $4.1-8.5$ \\
\hline 35 & 9 & 1.7 & $0.8-3.2$ & 1.2 & $0.6-2.4$ & 0 & 0 & $0.0-0.0$ & 0 & $0.0-0.0$ & 6 & 1.7 & $0.6-3.7$ & 1.2 & $0.4-2.6$ \\
\hline 39 & 9 & 1.7 & $0.8-3.2$ & 1.2 & $0.6-2.4$ & 0 & 0 & $0.0-0.0$ & 0 & $0.0-0.0$ & 6 & 1.7 & $0.6-3.7$ & 1.2 & $0.4-2.6$ \\
\hline 45 & 19 & 3.6 & $2.2-5.5$ & 2.6 & $1.6-4.1$ & 0 & 0 & $0.0-0.0$ & 0 & $0.0-0.0$ & 16 & 4.6 & $2.6-7.3$ & 3.2 & $1.8-5.2$ \\
\hline 51 & 21 & 3.9 & $2.5-6.0$ & 2.9 & $1.8-4.4$ & 0 & 0 & $0.0-0.0$ & 0 & $0.0-0.0$ & 16 & 4.6 & $2.6-7.3$ & 3.2 & $1.8-5.2$ \\
\hline 52 & 41 & 7.7 & 5.6-10.3 & 5.7 & $4.1-7.6$ & 0 & 0 & $0.0-0.0$ & 0 & $0.0-0.0$ & 26 & 7.4 & 4.9-10.7 & 5.2 & $3.4-7.5$ \\
\hline 56 & 14 & 2.6 & $1.4-4.4$ & 1.9 & $1.1-3.2$ & 0 & 0 & $0.0-0.0$ & 0 & $0.0-0.0$ & 12 & 3.4 & $1.8-5.9$ & 2.4 & $1.2-4.2$ \\
\hline 58 & 58 & 10.9 & $8.4-13.8$ & 8.0 & $6.2-10.3$ & 0 & 0 & $0.0-0.0$ & 0 & $0.0-0.0$ & 45 & 12.9 & $9.6-16.9$ & 9.0 & $6.7-11.9$ \\
\hline 59 & 15 & 2.8 & $1.6-4.6$ & 2.1 & $1.2-3.4$ & 0 & 0 & $0.0-0.0$ & 0 & $0.0-0.0$ & 14 & 4.0 & $2.2-6.6$ & 2.8 & $1.5-4.7$ \\
\hline 68 & 28 & 5.3 & $3.5-7.5$ & 3.9 & $2.6-5.6$ & 0 & 0 & $0.0-0.0$ & 0 & $0.0-0.0$ & 24 & 6.9 & $4.5-10.1$ & 4.8 & $3.1-7.1$ \\
\hline \multicolumn{16}{|l|}{ PHR-HPVs } \\
\hline 53 & 37 & 6.9 & 4.9-9.4 & 5.1 & $3.6-7.0$ & 1 & 5.3 & $0.1-26.0$ & 5.3 & $0.1-26.0$ & 32 & 9.2 & 6.4-12.7 & 6.4 & $4.4-8.9$ \\
\hline 66 & 32 & 6.0 & $4.1-8.4$ & 4.4 & $3.1-6.2$ & 0 & 0 & $0.0-0.0$ & 0 & $0.0-0.0$ & 30 & 8.6 & $5.9-12.0$ & 6.0 & $4.1-8.5$ \\
\hline \multicolumn{16}{|l|}{ LR-HPVs } \\
\hline 6 & 45 & 8.4 & $6.2-11.1$ & 6.2 & $4.6-8.3$ & 8 & 42.1 & $20.3-66.5$ & 42.1 & $20.3-66.5$ & 32 & 9.2 & $6.4-12.7$ & 6.4 & $4.4-8.9$ \\
\hline 11 & 31 & 5.8 & $4.0-8.2$ & 4.3 & $2.9-6.0$ & 5 & 26.3 & $9.1-51.2$ & 26.3 & $9.1-51.2$ & 20 & 5.7 & $3.5-8.7$ & 4.0 & $2.5-6.1$ \\
\hline 42 & 3 & 0.6 & $0.1-1.6$ & 0.4 & $0.1-1.2$ & 0 & 0 & $0.0-0.0$ & 0 & $0.0-0.0$ & 3 & 0.9 & $0.2-2.5$ & 0.6 & $0.1-1.7$ \\
\hline 43 & 1 & 0.2 & $0.0-1.0$ & 0.1 & $0.0-0.8$ & 0 & 0 & $0.0-0.0$ & 0 & $0.0-0.0$ & 1 & 0.3 & $0.0-1.6$ & 0.2 & $0.0-1.1$ \\
\hline 44 & 5 & 0.9 & $0.3-2.2$ & 0.7 & $0.2-1.6$ & 0 & 0 & $0.0-0.0$ & 0 & $0.0-0.0$ & 5 & 1.4 & $0.5-3.3$ & 1.0 & $0.3-2.3$ \\
\hline$x$ & 9 & 1.7 & $0.8-3.2$ & 1.2 & $0.6-2.4$ & 1 & 5.3 & $0.1-26.0$ & 5.3 & $0.1-26.0$ & 5 & 1.4 & $0.5-3.3$ & 1.0 & $0.3-2.3$ \\
\hline
\end{tabular}

$\mathrm{N}$ : total number of times which each genotype was detected.

* Percentages referred to the number of lesions infected by one or several genotypes (533 Total lesions, 19 benign lesions and 349 CIN 1).

** Percentages referred to the total number of virus detected (722 viruses in the Total of lesions, 19 in the Benign lesions and 499 in CIN 1 ).

C195\%: 95\% confidence intervals used for estimate percentages. 
Table 3 Distribution of HPV genotypes found in the study according to the pathological diagnosis

\begin{tabular}{|c|c|c|c|c|c|c|c|c|c|c|}
\hline \multirow{2}{*}{$\begin{array}{l}\text { Genotype } \\
\text { found }\end{array}$} & \multicolumn{5}{|c|}{ CIN 2-3 } & \multicolumn{5}{|c|}{ Invasive carcinoma } \\
\hline & $\mathrm{N}$ & $\% *$ & $\mathrm{Cl} 95 \%$ & $\% * *$ & $\mathrm{Cl} 95 \%$ & $\mathrm{~N}$ & $\% *$ & $\mathrm{Cl} 195 \%$ & $\% * *$ & $\mathrm{Cl} 95 \%$ \\
\hline \multicolumn{11}{|l|}{ HR-HPVs } \\
\hline 16 & 89 & 56.3 & $48.2-64.2$ & 45.2 & $38.1-52.4$ & 5 & 71.4 & $29.0-96.3$ & 71.4 & $29.0-96.3$ \\
\hline 18 & 9 & 5.7 & $2.6-10.5$ & 4.6 & $2.1-8.5$ & 1 & 14.3 & $0.4-57.9$ & 14.3 & $0.4-57.9$ \\
\hline 31 & 17 & 10.8 & $6.4-16.7$ & 8.6 & $5.1-13.5$ & 0 & 0 & $0.0-0.0$ & 0 & $0.0-0.0$ \\
\hline 33 & 14 & 8.9 & 4.9-14.4 & 7.1 & $3.9-11.6$ & 0 & 0 & $0.0-0.0$ & 0 & $0.0-0.0$ \\
\hline 35 & 3 & 1.9 & $0.4-5.4$ & 1.5 & $0.3-4.4$ & 0 & 0 & $0.0-0.0$ & 0 & $0.0-0.0$ \\
\hline 39 & 3 & 1.9 & $0.4-5.4$ & 1.5 & $0.3-4.4$ & 0 & 0 & $0.0-0.0$ & 0 & $0.0-0.0$ \\
\hline 45 & 3 & 1.9 & $0.4-5.4$ & 1.5 & $0.3-4.4$ & 0 & 0 & $0.0-0.0$ & 0 & $0.0-0.0$ \\
\hline 51 & 5 & 3.2 & $1.0-7.2$ & 2.5 & $0.8-5.8$ & 0 & 0 & $0.0-0.0$ & 0 & $0.0-0.0$ \\
\hline 52 & 15 & 9.5 & $5.4-15.2$ & 7.6 & $4.3-12.2$ & 0 & 0 & $0.0-0.0$ & 0 & $0.0-0.0$ \\
\hline 56 & 2 & 1.3 & $0.2-4.5$ & 1.0 & $0.1-3.6$ & 0 & 0 & $0.0-0.0$ & 0 & $0.0-0.0$ \\
\hline 58 & 13 & 8.2 & $4.5-13.7$ & 6.6 & $3.6-11.0$ & 0 & 0 & $0.0-0.0$ & 0 & $0.0-0.0$ \\
\hline 59 & 1 & 0.6 & $0.0-3.5$ & 0.5 & $0.0-2.8$ & 0 & 0 & $0.0-0.0$ & 0 & $0.0-0.0$ \\
\hline 68 & 4 & 2.5 & $0.7-6.4$ & 2.0 & $0.6-5.1$ & 0 & 0 & $0.0-0.0$ & 0 & $0.0-0.0$ \\
\hline \multicolumn{11}{|l|}{ PHR-HPVs } \\
\hline 53 & 4 & 2.5 & $0.7-6.4$ & 2.0 & $0.6-5.1$ & 0 & 0 & $0.0-0.0$ & 0 & $0.0-0.0$ \\
\hline 66 & 2 & 1.3 & $0.2-4.5$ & 1.0 & $0.1-3.6$ & 0 & 0 & $0.0-0.0$ & 0 & $0.0-0.0$ \\
\hline \multicolumn{11}{|l|}{ LR-HPVs } \\
\hline 6 & 5 & 3.2 & $1.0-7.2$ & 2.5 & $0.8-5.8$ & 0 & 0 & $0.0-0.0$ & 0 & $0.0-0.0$ \\
\hline 11 & 5 & 3.2 & $1.0-7.2$ & 2.5 & $0.8-5.8$ & 1 & 14.3 & $0.4-57.9$ & 14.3 & $0.4-57.9$ \\
\hline 42 & 0 & 0 & $0.0-0.0$ & 0 & $0.0-0.0$ & 0 & 0 & $0.0-0.0$ & 0 & $0.0-0.0$ \\
\hline 43 & 0 & 0 & $0.0-0.0$ & 0 & $0.0-0.0$ & 0 & 0 & $0.0-0.0$ & 0 & $0.0-0.0$ \\
\hline 44 & 0 & 0 & $0.0-0.0$ & 0 & $0.0-0.0$ & 0 & 0 & $0.0-0.0$ & 0 & $0.0-0.0$ \\
\hline$X$ & 3 & 1.9 & $0.4-5.4$ & 1.5 & $0.3-4.4$ & 0 & 0 & $0.0-0.0$ & 0 & $0.0-0.0$ \\
\hline
\end{tabular}

$\mathrm{N}$ : total number of times which each genotype was detected.

* Percentages referred to the number of lesions infected by one or several genotypes (158 CIN 2-3 and 7 invasive carcinomas).

** Percentages referred to the total number of virus detected (197 viruses in CIN 2-3 and 7 in Invasive carcinomas).

C195\%: 95\% confidence intervals used for estimate percentages.

found and in other case $(0.9 \%)$ six different genotypes were found.

The most common pattern of co-infection was double infection with HPV 16 and 58 (5 cases). Other patterns of co-infection were: HPV 16/31 (4 cases), HPV 16/53 (4 cases), HPV 16/18 (3 cases), and HPV 31/6 (3 cases).

In this kind of multiple infections, HPV 16 was the most common type (16.3\%) followed, in order of decreasing frequency, by HPV 31 (10.5\%), HPV 66 (9.7\%), HPV 58 (7.4\%), HPV 68 (7.4\%), HPV 53 (7.4\%), HPV 33 (6.2\%), HPV 52 (6.2\%), HPV 18 (4.7\%) and HPV 6 (4.7\%).

HR-HPVs were detected in $96.3 \%$ of CIN1 lesions with co-infection, PHR-HPVs in $37.4 \%$ and LR-HPVs in $15.9 \%$ of them. HPVs 16 and/or 18 were present in $46.7 \%$ of this cases and HPVs 6 and/or 11 in 15.0\% of them.

In CIN2-3 multiple infections cases, most co-infections consisted of two different genotypes (69.0\%). $27.6 \%$ of cases showed triple genotype infection and only $3.4 \%$ of them showed quadruple genotype infection.

The most common pattern of co-infection was double infection with HPV 16 and 52 (3 cases) and triple infection with HPV 16, 33 and 58 (2 cases).

In this kind of multiple infections, HPV 16 was the most common type (23.5\%) followed, in order of decreasing frequency, by HPV 33 (11.8\%), HPV 52 (11.8\%), HPV 58 (8.8\%), HPV 31 (7.4\%), HPV 68 (5.9\%), HPV 18 (4.4\%), HPV 45 (4.4\%), HPV 53 (4.4\%) and HPV 6 (4.4\%).

HR-HPVs were detected in 100\% of CIN2-3 lesions with co-infection, PHR-HPVs in $17.2 \%$ and LR-HPVs in $13.8 \%$ of them. HPVs 16 and/or 18 were present in $62.1 \%$ of this cases and HPVs 6 and/or 11 in $13.8 \%$ of them.

The data concerning co-infections are shown in Tables 4 and 5 . 
Table 4 Pathological diagnoses and co-infection occurrence

\begin{tabular}{|c|c|c|c|c|c|c|}
\hline \multirow{2}{*}{$\begin{array}{l}\text { Pathological } \\
\text { diagnosis }\end{array}$} & 2 HPV types & 3 HPV types & 4 HPV types & 5 HPV types & 6 HPV types & Total \\
\hline & $n^{\circ}(\%)$ & $n^{\circ}(\%)$ & $n^{\circ}(\%)$ & $n^{\circ}(\%)$ & $n^{\circ}(\%)$ & $n^{\circ}(\%)$ \\
\hline CIN 1 & 76 (71.0\%) & 22 (20.6\%) & 7 (6.5\%) & $1(0.9 \%)$ & $1(0.9 \%)$ & $107(78.7 \%)$ \\
\hline CIN 2-3 & $20(69.0 \%)$ & $8(27.6 \%)$ & $1(3.4 \%)$ & $0(0.0 \%)$ & $0(0.0 \%)$ & $29(21.3 \%)$ \\
\hline Total & $96(70.6 \%)$ & 30 (22.1\%) & $8(5.9 \%)$ & $1(0.7 \%)$ & $1(0.7 \%)$ & 136 (100\%) \\
\hline
\end{tabular}

Percentage referred to total of co-infections $(n=136)$.

\section{Discussion}

Of all the lesions, HPV 16 was the most frequent genotype. This finding is in accordance with many other studies carried out worldwide [20]. In our study, this genotype was present in $35.3 \%$ of total lesions. However, in a previous study carried in our hospital from 1993 to 2000, the HPV 16 presence in the total number of lesions was somewhat higher than at present (39\%) [21]. Also, in a recent study carried in a southern region of Spain from
2006 to 2007, this presence was even lower than ours (21.2\%) [17].

As expected, HPV16 presence increased in accordance with the grade of the lesion (15.8\% in benign lesions, 26.1\% in CIN1 cases, $56.3 \%$ in CIN2-3 cases and $71.4 \%$ in ICC).

HPV 31 was the second most frequent genotype in CIN1 lesions and in CIN2-3 lesions. In a study carried in an eastern region of Spain, HPV 31 was also the

Table 5 HPV genotype distribution in coinfection cases

\begin{tabular}{|c|c|c|c|c|c|c|c|c|c|c|}
\hline \multirow{2}{*}{$\begin{array}{l}\text { Genotype } \\
\text { found }\end{array}$} & \multicolumn{5}{|c|}{ CIN 1} & \multicolumn{5}{|c|}{ CIN 2-3 } \\
\hline & $\mathrm{N}$ & $\%$ * & Cl95\% & $\% * *$ & $\mathrm{Cl} 95 \%$ & $\mathrm{~N}$ & $\%$ * & $\mathrm{Cl} 95 \%$ & $\% * *$ & $\mathrm{Cl} 95 \%$ \\
\hline \multicolumn{11}{|l|}{ HR-HPVs } \\
\hline 16 & 42 & 39.3 & $30.0-49.2$ & 16.3 & $12.0-21.4$ & 16 & 55.2 & $35.7-73.6$ & 23.5 & $14.1-35.4$ \\
\hline 18 & 12 & 11.2 & 5.9-18.8 & 4.7 & $2.4-8.0$ & 3 & 10.3 & $2.2-27.4$ & 4.4 & $0.9-12.4$ \\
\hline 31 & 27 & 25.2 & $17.3-34.6$ & 10.5 & 7.0-14.9 & 5 & 17.2 & 5.8-35.8 & 7.4 & $2.4-16.3$ \\
\hline 33 & 16 & 15.0 & 8.8-23.1 & 6.2 & 3.6-9.9 & 8 & 27.6 & $12.7-47.2$ & 11.8 & $5.2-21.9$ \\
\hline 35 & 1 & 0.9 & $0.0-5.1$ & 0.4 & $0.0-2.1$ & 2 & 6.9 & $0.8-22.8$ & 2.9 & $0.4-10.2$ \\
\hline 39 & 3 & 2.8 & $0.6-8.0$ & 1.2 & $0.2-3.4$ & 1 & 3.4 & $0.1-17.8$ & 1.5 & $0.0-7.9$ \\
\hline 45 & 11 & 10.3 & $5.2-17.7$ & 4.3 & $2.2-7.5$ & 3 & 10.3 & $2.2-27.4$ & 4.4 & $0.9-12.4$ \\
\hline 51 & 11 & 10.3 & $5.2-17.7$ & 4.3 & $2.2-7.5$ & 1 & 3.4 & $0.1-17.8$ & 1.5 & $0.0-7.9$ \\
\hline 52 & 16 & 15.0 & $8.8-23.1$ & 6.2 & $3.6-9.9$ & 8 & 27.6 & $12.7-47.2$ & 11.8 & $5.2-21.9$ \\
\hline 56 & 6 & 5.6 & $2.1-11.8$ & 2.3 & $0.9-5.0$ & 1 & 3.4 & $0.1-17.8$ & 1.5 & $0.0-7.9$ \\
\hline 58 & 19 & 17.8 & $11.0-26.3$ & 7.4 & $4.5-11.3$ & 6 & 20.7 & $8.0-39.7$ & 8.8 & 3.3-18.2 \\
\hline 59 & 6 & 5.6 & $2.1-11.8$ & 2.3 & $0.9-5.0$ & 1 & 3.4 & $0.1-17.8$ & 1.5 & $0.0-7.9$ \\
\hline 68 & 19 & 17.8 & $11.0-26.3$ & 7.4 & $4.5-11.3$ & 4 & 13.8 & $3.9-31.7$ & 5.9 & $1.6-14.4$ \\
\hline \multicolumn{11}{|l|}{ PHR-HPVs } \\
\hline 53 & 19 & 17.8 & $11.0-26.3$ & 7.4 & $4.5-11.3$ & 3 & 10.3 & $2.2-27.4$ & 4.4 & $0.9-12.4$ \\
\hline 66 & 25 & 23.4 & $15.7-32.5$ & 9.7 & $6.4-14.0$ & 2 & 6.9 & $0.8-22.8$ & 2.9 & $0.4-10.2$ \\
\hline \multicolumn{11}{|l|}{ LR-HPVs } \\
\hline 6 & 12 & 11.2 & 5.9-18.8 & 4.7 & $2.4-8.0$ & 3 & 10.3 & $2.2-27.4$ & 4.4 & $0.9-12.4$ \\
\hline 11 & 5 & 4.7 & $1.5-10.6$ & 1.9 & $0.6-4.5$ & 1 & 3.4 & $0.1-17.8$ & 1.5 & $0.0-7.9$ \\
\hline 42 & 3 & 2.8 & $0.6-8.0$ & 1.2 & $0.2-3.4$ & 0 & 0 & $0.0-0.0$ & 0 & $0.0-0.0$ \\
\hline 43 & 1 & 0.9 & $0.0-5.1$ & 0.4 & $0.0-2.1$ & 0 & 0 & $0.0-0.0$ & 0 & $0.0-0.0$ \\
\hline 44 & 3 & 2.8 & $0.6-8.0$ & 1.2 & $0.2-3.4$ & 0 & 0 & $0.0-0.0$ & 0 & $0.0-0.0$ \\
\hline$x$ & 0 & 0 & $0.0-0.0$ & 0 & $0.0-0.0$ & 0 & 0 & $0.0-0.0$ & 0 & $0.0-0.0$ \\
\hline
\end{tabular}

$\mathrm{N}$ : total number of times which each genotype was detected.

* Percentages referred to the number of lesions infected by one or several genotypes (107 CIN 1 and 29 CIN 2-3 lesions).

**Percentages referred to the total number of virus detected in each kind of lesion (257 virus in CIN 1 and 68 in CIN 2-3 lesions).

C195\%: 95\% confidence intervals used for estimate percentages. 
second most frequent genotype in HSIL lesions and the presence found in these $(10.8 \%)$ was very similar to the presence obtained in our study (10.8\%) [19]. Previous meta-analysis reported the second position of HPV 31 in LSILs in Europe [11,20], and our results confirm this finding.

After HPV 16 and 31, HPV 58 was the third most common genotype found in our study. This event should be explained by the large number of immigrants who arrived in Spain over the past decade from Latin America, where this genotype is the second most frequently detected in HSIL after HPV 16 [13,19]. However, additional studies are needed to confirm this hypothesis. Moreover, the HPV 58 presence was somewhat higher in CIN1 cases (12.9\%) than in CIN2-3 cases (8.2\%), but this finding doesn't have statistical significance attending the $95 \%$ confidence intervals used for estimate these percentages (9.6-16.9 versus 4.5-13.7).

Our HPV 58 findings are in accordance with other recent study carried in a south-eastern region of Spain in which HPV 58 was the third most common genotype found and its frequency progressively decreased as lesions showed higher grades of dysplasia [22]. As some authors have reported, this decreasing HPV 58 frequency with the severity of the lesion may indicate that, despite the high frequency of HPV58 found in our area, LSIL caused by HPV58 would have less likelihood to progress to HSIL than a LSIL caused by HPV16 [22].

Globally, HPV 6 was the fourth most common genotype found in our study. Also, HPV 6 and 11 were the most frequent genotype in benign lesions (respectively, $42.1 \%$ and $26.3 \%$ ); but, surprisingly, HPV 16 was present in third position (15.8\%), as a single infection, in this type of lesions.

Some authors have reported that LR-HPV types -such as HPV 6 or HPV 11- are rarely identified as single infections in invasive cervical cancer [18]. In the previous study carried in our hospital, from 1993 to 2000, one case of single LR-HPV type infection in cervical cancer was reported [21]. Our current findings confirm that the detection of a single LR-HPV type (HPV 11) in ICC is a possible event. Further efforts are needed to understand in which conditions these HPV types can indeed induce invasive cervical cancer in rare circumstances.

In our study, the HPV 33 and 52 were the fifth and sixth most common types obtained. HPV 33 presence was basically no difference in CIN1 cases $(8.6 \%)$ than in CIN2-3 cases (8.9\%). This finding is in disagreement with the previously mentioned study carried in a southeastern region of Spain in which HPV 33 frequency increased in parallel with the severity of the lesion [22].

One previous meta-analysis reported the seventh position of HPV 52 in HSIL in Europe [20], although in our study HPV 52 was third in these types of lesions.
Therefore, the carcinogenic importance of this genotype is possibly increasing in our region at present.

Regarding the frequency of cases with the HPV 18 genotype is significant in our study that the frequency is not as high as the published in other international series. This data is in accordance with other published studies in Spain, in which HPV type 18 does not appear as a common type in the general population in our country [17]. Also, apparently, the HPV 18 presence was higher in CIN1 cases (7.2\%) than in CIN2-3 cases (5.7\%), but this finding doesn't have statistical significance attending the $95 \%$ confidence intervals used for estimate these percentages (4.7-10.4 versus 2.6-10.5).

As expected, HPV 16 and 18 presence was appreciably lower in CIN1 cases (32.4\%) than in CIN2-3 cases (61.4\%). However, in a study carried in a north-eastern region of Spain from 1999 to 2005, the joint frequency of these genotypes was considerably higher $(55 \%$ in CIN 1 cases, versus $80 \%$ in CIN 2 cases and $90 \%$ in CIN 3 cases) [16].

Moreover, in the earliest study carried in our hospital, from 1993 to 2000, the HPV 16/18 frequency was somewhat higher than in our current study (43\% in LSIL and $67 \%$ en HSIL) [21]. However, in a later study also carried in our hospital, the frequency of 16/18 HPV types was similar to our present study ( $41 \%$ in all types of lesions) [23]. Therefore, the joint frequency of these genotypes and its carcinogenic importance is possibly decreasing over time in our region.

A relationship was found between lesions and HRHPVs frequency. Thus, these genotypes were found more frequently in CIN2-3 cases (93.7\%) than in CIN1 cases (51.0\%) or benign lesions (21.1\%). The HR-HPVs presence found in our study in CIN2-3 cases (93.7\%) was similar to the frequency found in the previous studies carried in a region of northern Spain (88.1\%) and in a region in eastern Spain $(87.4 \%)[13,19]$.

Another issue worth mentioning is the co-infection occurrence, which seems to be more frequent in CIN1 cases (30.7\%) than in CIN2-3 cases (18.4\%). This finding is in accordance with the study carried in a south-eastern Spanish region mentioned above [22]; but it is at variance with the other previously mentioned study carried in a region in eastern Spain, in which the percentages of multiple infections were lower than in our study, and there were no considerable differences between groups of lesions (4.8\% in LSIL and 3.7\% in HSIL) [19].

In all types of lesions with multiple infections, the most common pattern of co-infection was double infection with HPV 16 and 58 (6 cases). The same result was found in the previously mentioned study carried in a region in northern Spain [13]. However, the most common pattern of co-infection in HSILs cases was double infection with HPV 16 y 52. It is unknown whether the association of 
these genotypes can produce a synergistic effect and increase carcinogenic risk. Further efforts are needed to clarify this hypothesis.

\section{Conclusion}

Regional variations in prevalence and distribution of HPV types have been verified in several epidemiological studies. In some countries such as Spain, these changes could be due to the arrival of a large number of women immigrants over the past decade. Furthermore, the generalized introduction of a HPV vaccination could produce an epidemiological pattern change and, consequently, in the coming years the majority of HPV infections could be produced by HPV types different from 16 and 18 .

As our study shows the current tetravalent vaccine could be effective in our geographical area for preventing all the invasive cervical carcinomas, however, the joint frequency of HPV 16/18 and its carcinogenic importance is possibly decreasing over time in our region. In addition, our study provides estimates of the important presence of other HR-HPV types - such as 31, 58, 33 and 52 - in different preneoplasic lesions. Thus, in the future the effectiveness of HPV vaccination in our geographical area and others with similar genotype distribution should be limited.

Therefore, the assessment of the distribution of HPV types associated with cervical cancer, in different geographic areas and over time, is necessary in order to apply the correct diagnostic and therapeutic measures in each region, to assess the impact of current vaccines and to guide the introduction of a new generation of them.

\section{Weaknesses of the study}

Our study presents some limitations. First, the origin of the samples from only one hospital may do hard to generalize the results to the entire population of Spain. However, this hospital is a reference centre in which patients of many districts and municipalities of the Region of Madrid are attended. Also, the results obtained in this study agree with data about population located in other Spanish regions and previously published.

Second, whereas more than 40 anogenital HPV types exist, only 20 were detected in this study. But all the HPVs genotypes usually implicated in the origin of the cervical cancer are detected.

Third, the small sample size of benign lesions and ICC affect to the study's significance. However, the difficulty to obtain ICC cases in Spain gives value to these results. In the future, it would be of interest to obtain more cases for further studies.

\section{Abbreviations}

HPV: Human papillomavirus; HR-HPV: High risk of carcinogenesis HPV type; PHR-HPV: Probably high risk of carcinogenesis HPV type; LR-HPV: Low risk of carcinogenesis HPV type; ICC: Invasive cervical carcinoma; CIN: Cervical intraepithelial neoplasia.

\section{Competing interests}

The authors declare that they have non-financial competing interests.

\section{Authors' contributions}

BGE participated in the design of the study, global data acquisition, analysis and preparation of the first draft. EMR revised critically the first and final draft, participated in the data acquisition, and conceived the design of the study, EAF conceptual and planning of the design of the study, surgical pathology data acquisition and analysis. All authors read and approved the final manuscript.

\section{Acknowledgments}

We would like to express our gratitude to Dolores Carneros for her technical assistance, to Jesús Esteban Hernández for his support to the statistical analysis and to Mrs. Rosalie Henderson Osborne (Universidad Rey Juan Carlos, Spain) for her contribution to the language revision and editorial assistance to this article.

\section{Author details}

${ }^{1}$ Department of Histology and Anatomical Pathology, Rey Juan Carlos University School of Medicine, Madrid, Spain. ${ }^{2}$ Department of Anatomical Pathology and Laboratories, Hospital General Universitario "Gregorio Marañón", Madrid, Spain. ${ }^{3}$ Universidad Rey Juan Carlos, Av de Atenas s/n., E28922 Alcorcón, Madrid, Spain.

Received: 28 April 2012 Accepted: 30 October 2012

Published: 20 November 2012

\section{References}

1. Schiffman M, Clifford G, Buonaguro FM: Classification of weakly carcinogenic human papillomavirus types: addressing the limits of epidemiology at the borderline. Infect Agent Cancer 2009, 4:8.

2. Pfister H: Human papillomaviruses and genital cancer. Avd Cancer Res 1987, 48:113-147.

3. De Villiers E-M, Fauquet C, Broker TR, Bernard H-U, zur Hausen H: Classification of papillomaviruses. Virology 2004, 324:17-27.

4. Muñoz N, Bosch FX, de Sanjosé S, Herrero R, Castellsagué X, Shah KV, Snijders PJ, Meijer CJ, International Agency for Research on Cancer Multicenter Cervical Cancer Study Group: Epidemiologic classification of human papillomavirus types associated with cervical cancer. N Engl J Med 2003, 348:518-527.

5. Plummer M, Schiffman M, Castle PE, Maucort-Boulch D, Wheeler CM: A 2-year prospective study of human papillomavirus persistence among women with a cytological diagnosis or atypical squamous cells of undetermined significance or low-grade squamous intraepithelial lesion. J Infect Dis 2007, 195:1582-1589.

6. Schiller JT, Lowy DR: Papillomavirus-like particles and HPV vaccine development. Semin Cancer Biol 1996, 7(6):373-382.

7. Zhow J, Sun XY, Stenzel DJ, Frazer $1 \mathrm{H}$ : Expression of vaccine recombinant HPV 16 L1 nd L2 ORF proteins in epithelial cells is sufficient for assembly of HPV virion-like particles. Virology 1991, 185(1):251-257.

8. Kirnbauer R, Chandrachud LM, O'Neil BW, Wagner ER, Grindlay GJ, Armstrong A, McGarvie GM, Schiller JT, Lowy DR, Campo MS: Virus-like particles of bovine papillomavirus type 4 in prophylactic and therapeutic immunization. Virology 1996, 219:37-44.

9. Conesa-Zamora P, Ortiz-Reina S, Moya-Biosca J, Doménech-Peris A, Orantes Casado FJ, Pérez-Gufillermo M, Egea-Cortines M: Genotype distribution of human papillomavirus (HPV) and co-infections in cervical cytologic specimens from two outpatient gynecological clinics in a region of southeast Spain. BMC Infect Dis 2009, 9:124.

10. Lehtinen M, Paavonen J, Wheeler CM, Jaisamrarn U, Garland SM, Castellsagué X, Skinner SR, Apter D, Naud P, Salmerón J, Chow SN, Kitchener H, Teixeira JC, Hedrick J, Limson G, Szarewski A, Romanowski B, Aoki FY, Schwarz TF, Poppe WA, De Carvalho NS, Germar MJ, Peters K, Mindel A, De Sutter P, Bosch FX, David MP, Descamps D, Struyf F, Dubin G, The HPV PATRICIA Study Group: Overall efficacy of HPV-16/18 AS04-adjuvanted vaccine against grade 3 or greater cervical intraepithelial neoplasia: 4-year end-of-study analysis of 
the randomised, double-blind PATRICIA trial. Lancet Oncol 2012, 13(1):89-99.

11. Clifford GM, Rana RK, Franceschi S, Smith JS, Gough G, Pimenta JM: Human papillomavirus genotype distribution in low-grade cervical lesions: comparison by geographic region and with cervical cancer. Cancer Epidemiol Biomarkers Prev 2005, 14(5):1157-1164.

12. De Sanjose S, Almiral R, Lloveras B, Font R, Diaz M, Muñoz N, Català I, Meijer CJ, Snijders PJ, Herrero R, Bosch FX: Cervical human papillomavirus infection in the female population in Barcelona, Spain. Sex Transm Dis 2003, 30:788-793.

13. De Oña M, Alvarez-Argüelles ME, Torrents M, Villa L, Rodriguez-Feijoo A, Palacio A, Boga JA, Tamargo A, Melón S: Prevalence, evolution, and features of infection with human papillomavirus: a 15-year longitudinal study of routine screening of a women population in the north of Spain. J Med Virol 2010, 82:597-604.

14. González C, Ortiz M, Canals J, Muñoz L, Jarrín I, de la Hera MG, García-Saiz A, del Amo J: Higher prevalence of human papillomavirus infection in migrant women from Latin America in Spain. Sex Transm Infect 2006, 82:260-262.

15. García-Espinosa B, Nieto-Bona MP, Rueda S, Silva-Sánchez LF, PiernasMorales MC, Carro-Campos P, Cortés-Lambea L, Moro-Rodríguez E: Genotype distribution of cervical human papillomavirus DNA in women with cervical lesions in Bioko, Ecuatorial Guinea. Diagn Pathol 2009, 4:31.

16. Bernal M, Burillo I, Mayordomo Jl, Moros M, Benito R, Gil J: Human papillomavirus (HPV) infection and intraepithelial neoplasia and invasive cancer of the uterine cervix: a case-control study in Zaragoza, Spain. Infect Agent Cancer 2008, 3:8.

17. Cobo F, Concha A, Ortiz M: Human papillomavirus (HPV) type distribution in females with abnormal cervical cytology. A correlation with histological study. Open Virol J 2009, 3:60-66.

18. De Sanjose S, Quint WG, Alemany L, Geraets DT, Klaustermeier JE, Lloveras B, Tous S, Felix A, Bravo LE, Shin HR, Vallejos CS, de Ruiz PA, Lima MA, Guimera N, Clavero O, Alejo M, Llombart-Bosch A, Cheng-Yang C, Tatti SA, Kasamatsu E, Iljazovic E, Odida M, Prado R, Seoud M, Grce M, Usubutun A, Jain A, Suarez GA, Lombardi LE, Banjo A, Menéndez C, Domingo EJ, Velasco J, Nessa A, Chichareon SC, Qiao YL, Lerma E, Garland SM, Sasagawa T, Ferrera A, Hammouda D, Mariani L, Pelayo A, Steiner I, Oliva E, Meijer CJ, Al-Jassar WF, Cruz E, Wright TC, Puras A, Llave CL, Tzardi M, Agorastos T, Garcia-Barriola V, Clavel C, Ordi J, Andújar M, Castellsagué X, Sánchez Gl, Nowakowski AM, Bornstein J, Muñoz N, Bosch FX, Retrospective International Survey and HPV Time Trends Study Group: Human papillomavirus genotype attribution in invasive cervical cancer: a retrospective cross-sectional worldwide study. Lancet Oncol 2010, 11(11):1048-1056.

19. García-García JA, Pérez-Vallés A, Martorell M, Gómez B, Gómez-Cabrero D, Soler F, Calabuig C: Distribution of human papillomavirus types in women from Valencia, Spain, with abnormal cytology. Acta Cytol 2010, 54:159-164.

20. Clifford G, Franceschi S, Diaz M, Muñoz N, Villa LL: Chapter 3: HPV typedistribution in women with and without cervical neoplastic diseases. Vaccine 2006, 24(S3):26-34.

21. Lacruz Pelea C, Di Martino Ortiz B, Álvarez Fernández ÁFE: Incidencia de los diferentes tipos de papiloma virus humano (HPV) en las lesiones escamosas del cérvix uterino. Rev Es Patol 2003, 36(1):79-84.

22. Doménech-Peris A, Conesa-Zamora P, Sahuquillo-Frias L, Ortiz-Reina S, Moya Biosca J, Acosta-Ortega J, Pérez Guillermo M, Egea-Cortines M: Human papillomavirus genotyping in histological sections of precursor lesions of cervical carcinoma: its role as a possible adjunct for the evaluation of the oncogenic potential of specific human papillomavirus genotypes - a study in a coastal region of Southeastern Spain. Gynecol Obstet Invest 2010, 70:113-119.

23. Lacruz Pelea C, Muñoz Fernández de Legaria M: Vacunas VPH: lesiones de cérvix y tipos virales asociados en la Comunidad de Madrid. Rev Es Patol 2009, 42:121-125.

doi:10.1186/1471-2407-12-533

Cite this article as: García-Espinosa et al:: Genotype distribution of human papillomavirus (HPV) in histological sections of cervical intraepithelial neoplasia and invasive cervical carcinoma in Madrid, Spain. BMC Cancer 2012 12:533.

\section{Submit your next manuscript to BioMed Central and take full advantage of:}

- Convenient online submission

- Thorough peer review

- No space constraints or color figure charges

- Immediate publication on acceptance

- Inclusion in PubMed, CAS, Scopus and Google Scholar

- Research which is freely available for redistribution 